\title{
Interesting Response with the Delivery of Right Ventricular Extrastimulus of Increasing Prematurity
}

\author{
KRISHNA KUMAR MOHANAN NAIR, MBBS, MD, DM, ${ }^{1}$ NARAYANAN NAMBOODIRI, MBBS, MD, DM, ${ }^{1}$ \\ and AJITKUMAR VALAPARAMBIL, MBBS, MD, DM ${ }^{1}$
}

'Department of Cardiology, Sree Chitra Tirunal Institute for Medical Sciences and Technology, Thiruvananthapuram, Kerala, India

\begin{abstract}
A 45-year-old man with no structural heart disease underwent an electrophysiology study for recurrent episodes of palpitation. There was no evidence of pre-excitation on the baseline electrocardiogram. Baseline intervals, including the $A-H$ and $H-V$ intervals, were within the normal limits and remained so during the electrophysiology study. An interesting response was observed with the delivery of ventricular extrastimuli with increasing prematurity from the right ventricular apex, triggering consideration of the possible mechanism.
\end{abstract}

KEYWORDS. Retrograde conduction, right bundle branch block, ventricular extrastimulus.
ISSN 2156-3977 (print) ISSN 2156-3993 (online) CC BY 4.0 license

(C) 2021 Innovations in Cardiac Rhythm Management
A 45-year-old man with no structural heart disease underwent an electrophysiology study for recurrent episodes of palpitation. There was no evidence of pre-excitation on the baseline electrocardiogram (ECG) and baseline intervals, including the $\mathrm{A}-\mathrm{H}$ and $\mathrm{H}-\mathrm{V}$ intervals, were within the normal limits. During the electrophysiology study, these intervals remained within normal limits. However, ventricular extrastimuli (VES) delivered with increasing prematurity from the right ventricular (RV) apex led to an interesting response.

Figure 1A shows VES delivered after a drive train of eight beats with a pacing cycle length of $450 \mathrm{~ms}$ at a prematurity of $280 \mathrm{~ms}$. In response, the V-H and V-A intervals measured $60 \mathrm{~ms}$ and $105 \mathrm{~ms}$, respectively. Meanwhile, with a VES of $260 \mathrm{~ms}$, the $\mathrm{V}-\mathrm{H}$ and $\mathrm{V}-\mathrm{A}$ intervals increased to $110 \mathrm{~ms}$ and $155 \mathrm{~ms}$, respectively (Figure 1B). The atrial

The authors report no conflicts of interest for the published content. Manuscript received July 1, 2020. Final version accepted December 28, 2020.

Address correspondence to: Krishna Kumar Mohanan Nair, MBBS, MD, DM, Department of Cardiology, Sree Chitra Tirunal Institute for Medical Sciences and Technology, Thiruvananthapuram, Kerala 695011, India. Email: kakkam@gmail.com. activation pattern remained concentric with both coupling intervals, and the $\mathrm{H}-\mathrm{A}$ intervals were $45 \mathrm{~ms}$ with both coupling intervals.

\section{Discussion}

An increase in the $\mathrm{V}-\mathrm{A}$ interval following an increase in the prematurity of the VES may be (1) due to a sudden shift in retrograde conduction from a fast atrioventricular (AV) nodal pathway to a slow AV nodal pathway, (2) the result of accessory pathway conduction, or (3) a consequence of retrograde right bundle branch block (RBBB). With the development of retrograde RBBB, retrograde conduction occurs via transseptal activation and retrograde conduction through the left bundle in order to reach the His bundle. This is suggested by an increase in the $\mathrm{V}-\mathrm{H}$ interval of at least $50 \mathrm{~ms}$ in response to VES or a $\mathrm{V}-\mathrm{H}$ jump. ${ }^{1}$

In the index case, in response to an increase in the prematurity of the VES by $20 \mathrm{~ms}$, an increase in the V-A interval by $50 \mathrm{~ms}$ was preceded by the $\mathrm{V}-\mathrm{H}$ jump. This suggests not only the development of retrograde RBBB but also retrograde nodal conduction as the increments in the 


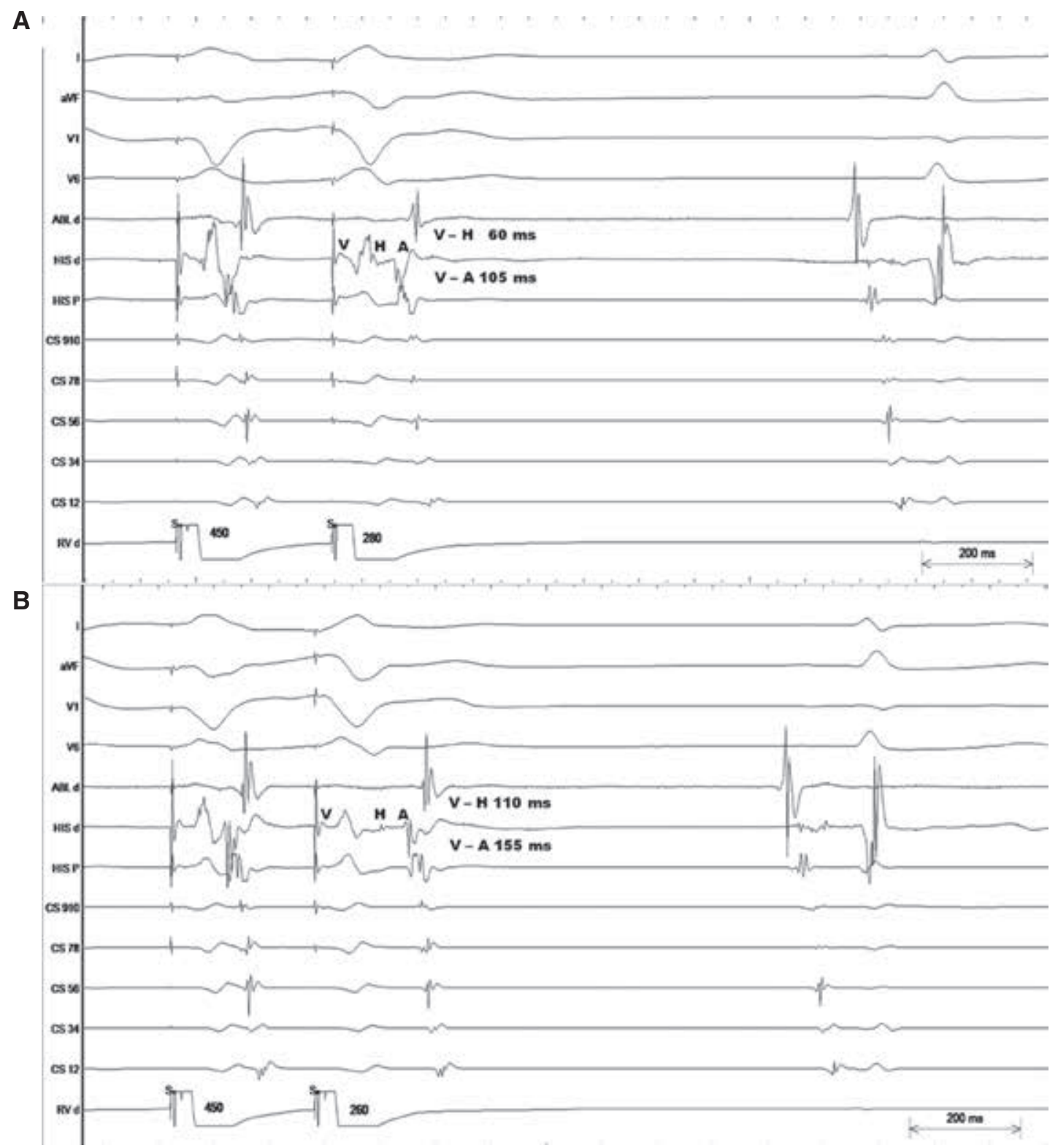

Figure 1: A: Represents surface ECG (I, aVF, V1, V6) and intracardiac electrograms at mapping and ablation catheter distal at the right atrial appendage ( $A B L d$ ), His-bundle electrogram distal (HIS d), His-bundle electrogram proximal (HIS p), coronary sinus (CS) 9,10 dipoles at CS ostium, CS 1,2 dipoles at distal CS, and RV apex distal (RV d) showing a response to VES delivered at a prematurity of 280 ms. B: Surface ECG (I, aVF, V1, V6) and intracardiac electrograms at mapping and ablation catheter distal at the right atrial appendage ( $A B L d$ ), His bundle electrogram distal (HIS d), His bundle electrogram proximal (HIS p), CS 9,10 dipoles at CS ostium, CS 1,2 dipoles at distal CS, and RV apex distal (RV d) showing response to VES delivered at a prematurity of 260 ms.

$\mathrm{V}-\mathrm{H}$ interval and the $\mathrm{V}-\mathrm{A}$ interval were identical to one another. With exclusive retrograde nodal conduction, the His bundle will be activated invariably prior to the AV node and, hence, the development of retrograde RBBB will prolong the $\mathrm{V}-\mathrm{A}$ interval. The increment in the $\mathrm{V}-\mathrm{A}$ interval will be at least as much as the increase in the $\mathrm{V}-\mathrm{H}$ interval. When the retrograde conduction is extranodal or via the accessory pathway, despite the development of retrograde $R B B B$, there will be no change in the $V-A$ interval.
The trace shows the development of retrograde RBBB development followed by retrograde nodal conduction in response to VES of increasing prematurity.

\section{Reference}

1. Kapa S, Henz BD, Dib C, et al. Utilization of retrograde right bundle branch block to differentiate atrioventricular nodal from accessory pathway conduction. J Cardiovasc Electrophysiol. 2009;20(7):751-758. 